\title{
Multidetector computed tomography: a corner stone imaging modality in evaluation of acute small bowel diseases
}

\author{
Doaa M. Emara ${ }^{{ }^{*}}$ (D), Khaled M. Moghazy ${ }^{1}$, Galal M. Abouelnagah ${ }^{2}$ and Ahmed H. Amer ${ }^{1}$
}

\begin{abstract}
Background: The diagnosis of acute small bowel diseases is one of the challenging issues that confronted by the radiologists so accurate diagnosis is essential to determine the appropriate way of management. CT has become the preferred imaging tool to evaluate acute small bowel diseases. Our study aimed to assess the role of MDCT in evaluation of acute abdomen secondary to small bowel origin by identification and differentiation between different acute small bowel pathologies.

Results: Thirty-eight patients presented with acute abdomen of small bowel origin from June 2019 to September 2019. The mean age of incidence was $48 \pm 19$ years ranged from 4 to 88 years. Males represented by 23 patients (60.5\%). Acute exacerbation of inflammatory bowel diseases (Crohn's disease) represented by (34.2\%), small bowel obstruction (31.6\%), ischemic bowel diseases (21.1\%), small bowel perforation (10.5\%) and infectious (TB enteritis) small bowel disease (2.6\%). MDCT had an overall high sensitivity (97.3\%) in assessment of acute small bowel diseases in correlation with post-operative data and follow-up response to management.
\end{abstract}

Conclusions: MDCT is a reliable diagnostic imaging tool for assessment of patients with acute abdomen secondary to small bowel origin with high-efficiency in differentiation between different pathological entities that causing acute abdomen.

Keywords: Multidetector computed tomography (MDCT), Acute abdomen, Acute small bowel diseases, Crohn's disease, Small bowel obstruction, Ischemic bowel diseases, Small bowel perforation and TB enteritis

\section{Background}

The small bowel loops have unique vascular supply, specific anatomical considerations and different physiological functions making them associated with pathological entities different from those affecting the large bowel loops [1].

The patients with acute small bowel insult have different presentations and symptoms according to the underlying small bowel disease, usually diffuse non-localized abdominal pain is the first presentation leading to

\footnotetext{
*Correspondence: dr.emara_doaa@yahoo.com; dr.emara-doaa@hotmail.com ${ }^{1}$ Department of Radiodiagnosis, Faculty of Medicine, University of Alexandria, Alexandria, Egypt

Full list of author information is available at the end of the article
}

utilization of different imaging modalities to assess the underlying disease $[1,2]$. Intravenous contrast-enhanced CT represents the corner stone imaging modality for assessment of acute small bowel diseases and for differentiation between acute abdomen of small bowel origin and other causes of acute abdomen [2,3].

Multidetector CT provides good spatial and contrast resolution, multiplanar reconstructions and more efficient scanning with few or no artifacts. Multidetector $\mathrm{CT}$ provides detailed examination of the small bowel diseases $[1,4]$.

The clinical suspicious and appropriate selection of multidetector CT protocols can be implemented to enhance diagnostic accuracy of small bowel diseases according to the pathology $[1,5]$. 
Multidetector CT scan help in diagnosis of mesenteric ischemia, identify its underlying causes, extension and severity $[2,6]$.

Multidetector CT enterography plays an important role in imaging of inflammatory bowel disease (IBD), including assessment of the disease activity and extraluminal acute complications $[7,8]$.

If there is clinical suspicion for a neither vascular nor inflammatory bowel disease (IBD), routine intravenous contrast-enhanced CT of the abdomen and pelvis with multiplanar reconstructions has important role in diagnostic evaluation of small bowel obstruction and small intestinal perforation [1].

\section{Aim of the study}

The aim of this study was to assess the role of multidetector computed tomography in assessment of cases with acute abdomen secondary to small bowel diseases.

\section{Methods}

\section{Patients}

A prospective study included 38 patients suffering from acute abdominal symptoms secondary to suspected acute small intestinal disease had been assessed by multidetector computed tomography (MDCT) between June 2019 and September 2019 at our institute. The final diagnosis was confirmed by pathological correlation, post-operative data and clinical response to medical treatment.

Inclusion criteria:

Patients with acute abdomen secondary to small bowel diseases.

\section{Exclusion criteria:}

- Acute abdomen due to other causes than acute small bowel diseases.

- Chronic small bowel diseases.

- Patients have contraindications from intravenous contrast materials, e.g., patient with renal impairment and sever reaction to contrast materials.

\section{Methods}

Multidetector computed tomography examination of the abdomen and pelvis after taking detailed history and clinical evaluation including:

- Routine CT abdomen and pelvis with contrast.

- CT entero-colonography if indicated (done for 22 patients; for patients suspected to have acute exacerbation of Crohn's disease and patients suspected to have ischemic small bowel disease).
- MDCT was performed using 64 slice/rotation Philips CT machines (Philips health care Nederland B.V). Patients scanned in supine position without gantry tilt. The CT protocol consisted of volumetric data acquisition commencing at the level of the diaphragm to the symphysis pubis with breath-hold technique (as possible as patient can) to avoid the motion artifacts. The scanning parameters used were: KVP: $120-140 \mathrm{mAs}$ : $150-450$, tube rotation time: $0.33 \mathrm{~s} /$ rotation, slice thickness: $0.5-1 \mathrm{~mm}$, collimation: $6 \mathrm{~mm}$ and reconstruction interval: $1 \mathrm{~mm}$.

- No special preparation was needed except fasting for about 4-6 h before examination for patient having small bowel obstruction due to the presence of dilated bowel loops.

- For CT enterography technique: The cleansing preparation requires a low residue diet, ample fluids and fasting six hours prior to the examination. Patients drank $500 \mathrm{~mL}$ aliquots of iso-osmotic mannitol (mannitol 10\% diluted with water in a ratio of 1:1 in order to be iso-osmotic, i.e., $1 \mathrm{~L}$ mannitol $10 \%$ and $1 \mathrm{~L}$ water) in $60 \mathrm{~min}$ for a total of $2000 \mathrm{~mL}$. The total amount of fluid was given through $1 \mathrm{~h}$ at 15, 30, 45 and $60 \mathrm{~min}$.

- Enema (neutral contrast) used in cases did CT entero-colonography for 21 patients.

- Pre- and post-contrast CT examination done for all patients, all post-contrast images were obtained in portal venous phase at $70 \mathrm{~s}$, in cases with suspected vascular compromise and inflammatory bowel diseases: post-contrast images obtained in arterial phase [30 s] and in portal venous phases (70 s). Non-ionic iodinated contrast material (omnipaque 350) was injected with a power injector at rate $3 \mathrm{~mL} / \mathrm{s}$ through the antecubital vein. The total amount of injected contrast material was injected according to the body weight of the patient $(1.5 \mathrm{~mL} / \mathrm{kg}, 350 \mathrm{mg} / \mathrm{mL})$.

\section{Data analysis and interpretation}

- The MDCT images were interpreted using dedicated workstation and post-processing software using multiplanner reconstruction (axial, sagittal and coronal views), MIP (maximum intensity projection) and curved MPR (multiplanner reformatted images).

- Data were coded and entered using the statistical package for the Social Sciences (SPSS) version 25 (IBM Corp., Armonk, NY, USA). The data were summarized to describe quantitative variables and frequencies (number of cases) and percentages for each categorical variable. 
- Diagnostic analysis was used to assess the role of MDCT in evaluation of acute small bowel diseases related to acute abdominal pain.

- The CT data and sensitivity were correlated with the pathological (13 patients), surgical (16 patients) and medical results (8 patients) to reach the final diagnosis. The rest of patients are already known patient with infectious bowel disease (TB) the role of CT was to assess the involvement and extension of the pathological process.

\section{Results}

The current study included 38 patients: 23 males and 15 females. The age of the involved cases ranges from 4 to 88 years with mean age of incidence at the 5 th decade (48 years).

- Distribution of the studied cases according to the final diagnosis:

Acute exacerbation of inflammatory bowel diseases (Crohn's disease) representing the most frequent cause of acute abdomen secondary to small bowel diseases by involving about $34.2 \%$ of the patient sample, small bowel obstruction representing $31.6 \%$, ischemic bowel disease representing $21.1 \%$, small bowel perforation representing $10.5 \%$ and finally infectious small bowel disease (TB enteritis) representing $2.6 \%$ of the examined patients (Fig. 1).
- Acute exacerbation of inflammatory small bowel diseases:

Thirteen patients with history of pathologically proved Crohn's disease are suspected to have acute exacerbation of inflammatory bowel disease, 12 patients showed CT signs of acute exacerbation while one patient showed no sign of activity (false negative result) as the histopathological assessment showed active ileitis (Fig. 2). So, the estimated sensitivity of CT entero-colonography for detection of activity of Crohn's disease through the examined patients is about $92.3 \%$.

CT features of acute exacerbation of Crohn's disease (Table 1):

1. The most frequent $\mathrm{CT}$ features of acute exacerbation of Crohn's disease are mural thickening with mucosal hyperenhancement and submucosal edema (mural stratification) as well as surrounding fat stranding, which seen in 12 patients. The most common site of activity is terminal ileum which presented in 10 patients (Fig. 2).

2. Comb sign is the second frequent sign of activity, which seen in 11 patients (Fig. 2).

3. Reactive mesenteric lymph nodes are the third frequent sign, which seen in 10 patients (Fig. 2).

4. Complications of acute exacerbation include fistulae, abscess formation and inflammatory stricture:

(a) Fistulae are showed in 4 patients; 2 patients showed entero-cutaneous fistulae (Fig. 2), one

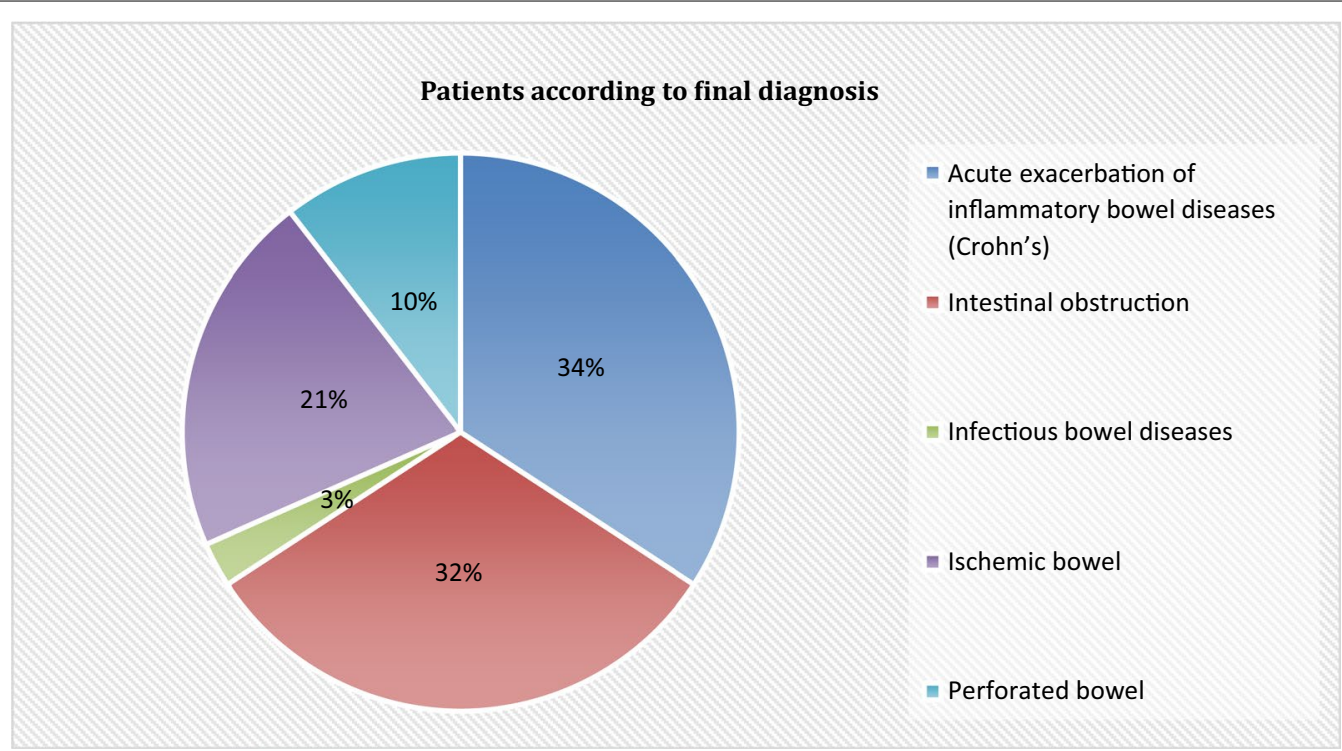

Fig. 1 Distribution of studies cases according to final diagnosis 


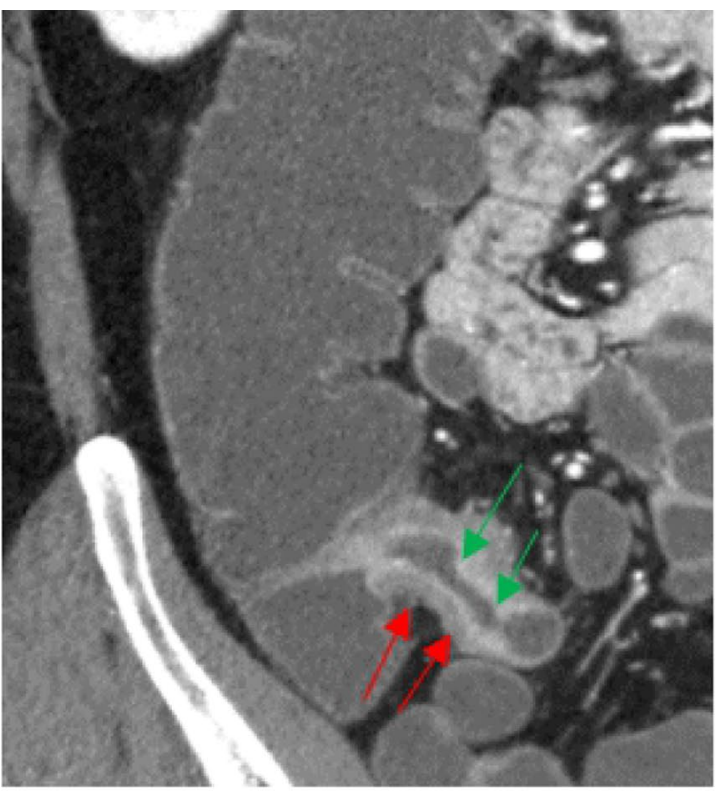

(A)

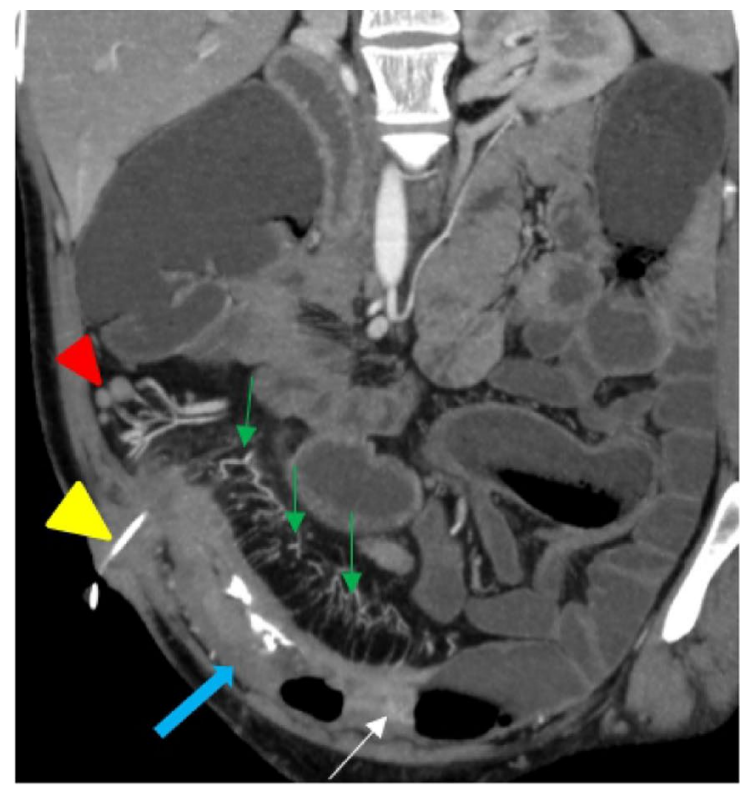

(B)

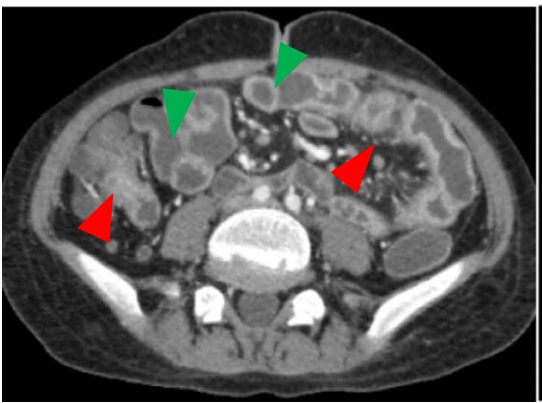

(C)

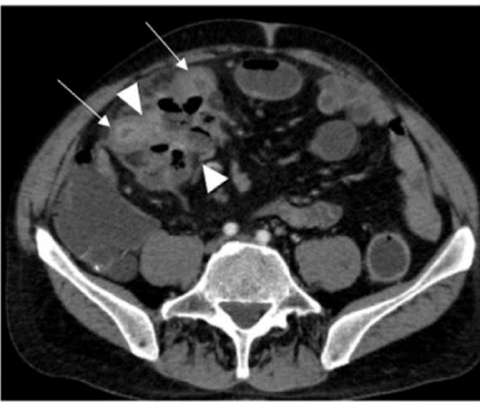

(D)

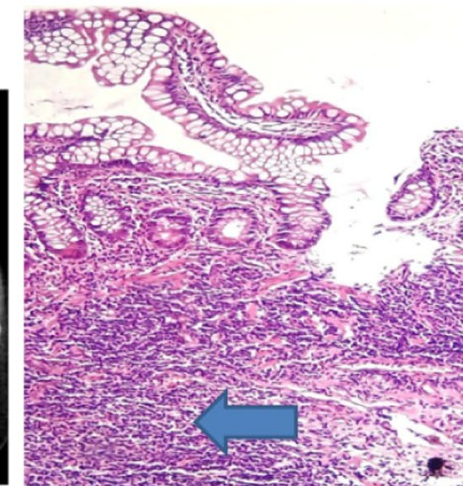

(E)

Fig. 2 Different patients with history of pathologically proved Crohn's disease. A Coronal CT in arterial phase showing diffuse mural thickening at the terminal ileum with mucosal hyperenhancement (green arrows) and submucosal edema (red arrows). B CT coronal MIP in arterial phase showing terminal ileal mural thickening with mucosal hyperenhancement (white arrow) and submucosal edema (blue arrow), surrounding congested vasa recta 'comb sign' (green arrows) and few reactive lymph nodes (red arrow head) with entero-cutaneous fistula (yellow arrow head) ending in terminal ilium after injection of positive contrast through the fistulous tract. C Axial CT in arterial phase showing small bowel skip lesions (red arrow heads) in between apparently normal bowel loops (green arrow heads). D Axial CT in arterial phase shows abscess formation (white arrow heads) in between inflamed ileal loops (white arrows). E Histopathology image showing focal mucosal ulceration with lymphocytic infiltration involving mucosa and submucosa (arrow)

patient showed entero-cutaneo-vesical and the other showed entero-enteric fistula.

(b) Abscess formation presented in 3 patients (Fig. 2) as well as mild inflammatory stricture coupled with proximal bowel dilatation demonstrated in 3 patients.

5. Skip lesions in different loops demonstrated in 3 patients (Fig. 2), while the rest of patients showing only single affected bowel segment.
6. Bilateral sacroiliitis is the only extra-intestinal CT features along the examined Crohn's disease patients which presented in 2 patients.

- Infectious small bowel diseases:

Only presented in one case in this study with known history of TB infection, the patient presented with acute abdominal pain, fever, anorexia and weight loss. CT 
Table 1 Distribution of the studied cases according to CT findings for acute exacerbation of inflammatory bowel diseases (Crohn's) $(n=13)$

\begin{tabular}{lcc}
\hline CT findings & No & $\%$ \\
\hline Mucosal hyperenhancement & 12 & 92.3 \\
Mural thickening & 12 & 92.3 \\
Terminal ileum & 10 & 83.3 \\
lleum and jejunum & 1 & 8.3 \\
Duodenum ileum and jejunum & 1 & 8.3 \\
Submucosal edema & 12 & 92.3 \\
Fat stranding & 12 & 92.3 \\
Comb sign & 11 & 84.6 \\
Enlarged LN & 10 & 76.9 \\
Fistula & 4 & 30.7 \\
Skip lesions & 3 & 23.1 \\
Abscess formation & 3 & 23.1 \\
Dilated bowel loops & 3 & 23.1 \\
Extra-intestinal manifestations (Bilateral & 2 & 15.4 \\
sacroiliitis) & & \\
\hline
\end{tabular}

features of the presented patient are characterized by distal ileal bowel loops and cecum mild mural thickening with mucosal hyperenhancement, no mural stratification noted, this is coupled with necrotic mesenteric lymph nodes which is characteristic CT features for TB enteritis (Fig. 3).

\section{- Intestinal obstruction:}

Twelve patients diagnosed with intestinal obstruction; most of patient presented with acute abdominal pain, vomiting and constipation.
The causes of small bowel obstruction include adhesive bowel obstruction which is the most common frequent cause of intestinal obstruction among the examined patients, while the obstructed hernia, intussusception and gall stone ileus are equally presented. Seven patients of adhesive bowel obstruction (Fig. 4) are surgically proved while only one patient managed by conservative treatment and follow up who shows complete resolution of symptoms after one day of conservative treatment. Only 2 patients of adhesive small bowel obstruction have no history of previous surgical operation, while the rest of patients have history of previous surgical intervention. One patient diagnosed by IO secondary to combination of intussusception and adhesive bowel obstruction. One patient showing recurrent obstructed para-umbilical hernia (Fig. 4). One patient diagnosed by IO secondary to intussusception (Fig. 4) with a leading point (Meckel's diverticulum). Gall stone ileus (Fig. 4) was diagnosed as cause of bowel obstruction in one patient.

\section{- Ischemic bowel disease:}

Eight patients diagnosed with mesenteric bowel ischemia; the causes of small bowel ischemia secondary to mesenteric vascular occlusion include SMV occlusion (Fig. 5) which is the most frequent cause of ischemic bowel disease among the examined patient. Five patients are diagnosed with SMV occlusion. Two patients are managed surgically, while the rest of them are managed conservatively. SMA occlusion (Fig. 5) presented in three patients; one of them diagnosed with partial thrombosis of the superior mesenteric artery and managed conservatively, while the other one showing complete occlusion of the superior mesenteric artery associated with occlusion

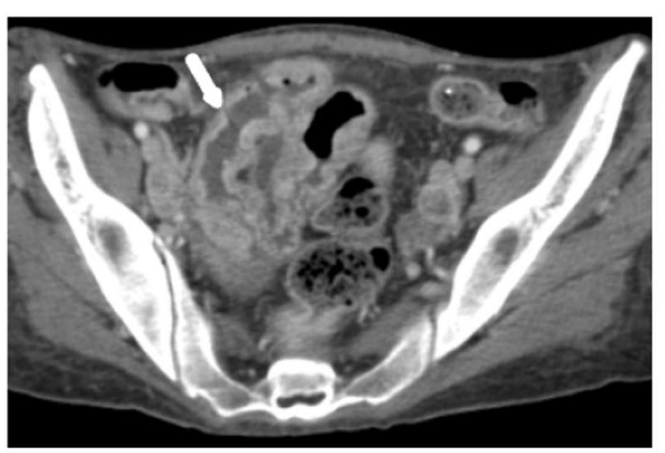

(A)

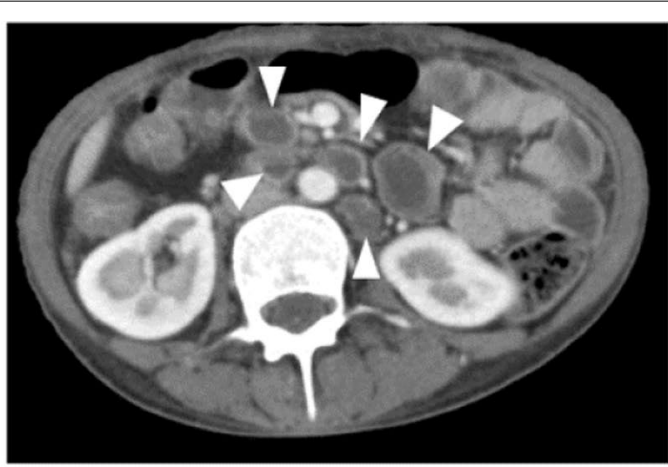

(B)

Fig. 3 A 35-year-old female patient with a given history of TB infection complaining of acute abdominal pain, fever anorexia and weight loss. A Axial CT in venous phase shows mild mural thickening with mucosal hyperenhancement (white arrow). B Axial CT in venous phase shows multiple para-aortic and mesenteric necrotic lymph nodes (arrow heads) 


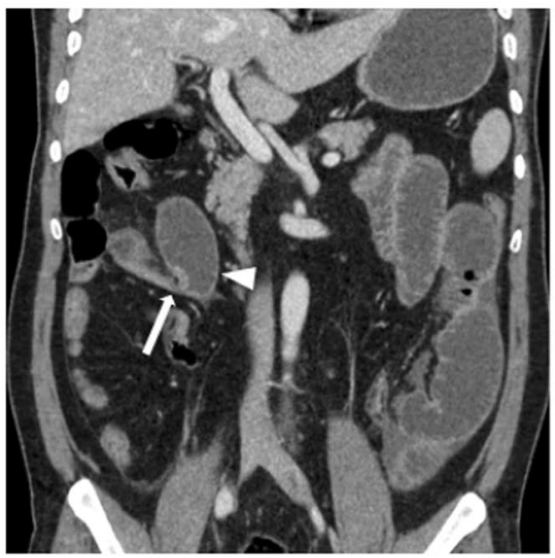

(A)

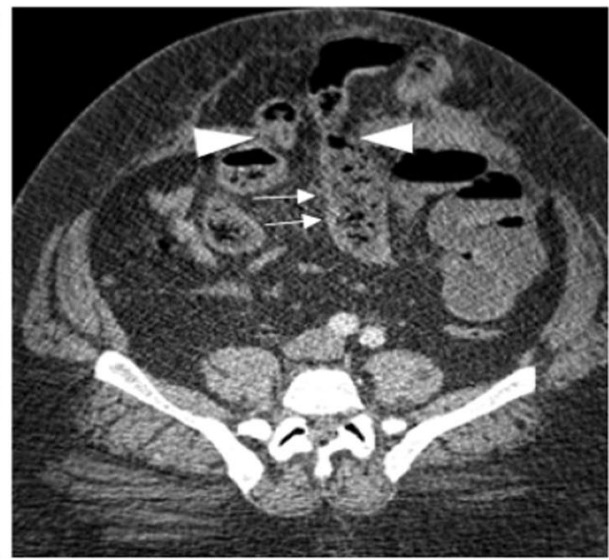

(B)

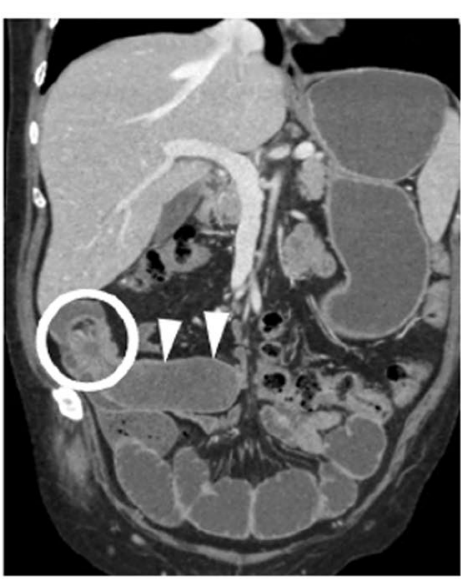

(C)

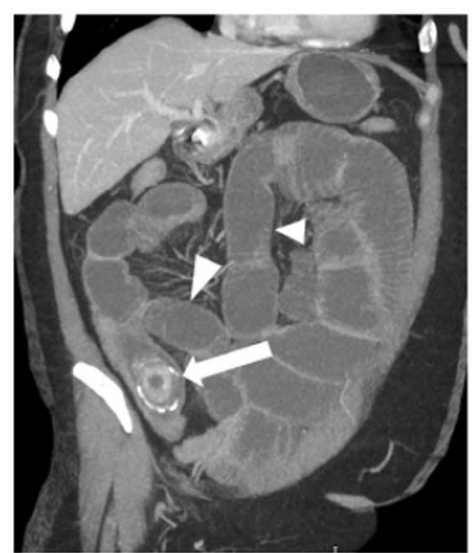

(D)

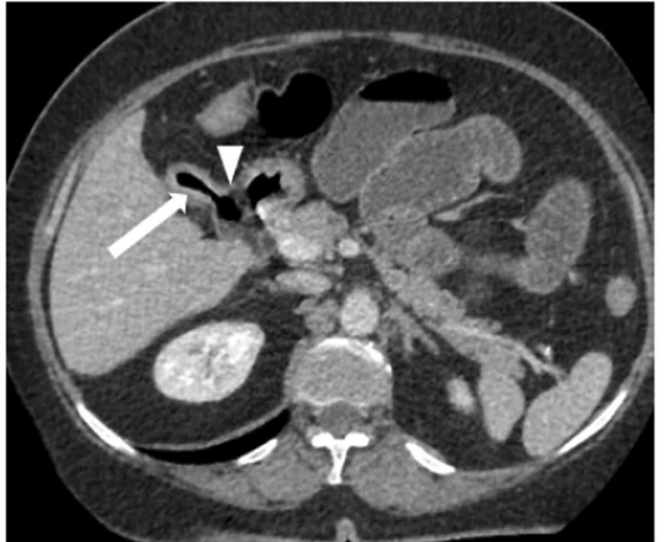

(E)

Fig. 4 Different patient diagnosed with small bowel obstruction presented with acute abdominal pain, vomiting and constipation. A Coronal CT in venous phase for patient diagnosed with adhesive intestinal obstruction showing proximally dilated bowel loops (white arrow head) and the site of transition zone of adhesive band (white arrow). B Axial CT in arterial phase for patient diagnosed with recurrent obstructed para-umbilical hernia showing hernial defect (white arrow head) and proximal dilated bowel loops showing intestinal feces sign (white arrow). C Coronal oblique CT in venous phase with MPR for patient diagnosed with intussusception showing the site of intussuscepted bowel loop (white circle) with the leading point, dilated Meckel's diverticulum (white arrow heads). D Coronal oblique view CT in venous phase with MPR and MIP for patient diagnosed with gall stone ileus showing the hyperdense gall stone within small bowel loops (white arrow) and proximal dilated bowel loops (white arrow heads). E Axial CT in venous phase for patient diagnosed with gall stone ileus showing gall bladder pneumobilia (white arrow) and cholecysto-duodenal fistula (white arrow head)

of both renal arteries with subsequent renal infarction, unfortunately the patient died. The third patient shows thrombosis of one of the superior mesenteric artery branches which was managed surgically.

CT features of bowel ischemia include mural thickening (Fig. 5) which is the most frequent CT findings of ischemic bowel diseases among the examined patients. Surrounding fat stranding (Fig. 5) is the second frequent $\mathrm{CT}$ features of small bowel ischemia which presented in 5 patients. Only two patients showing mucosal hypoenhancement which is a prognostic sign for irreversible bowel ischemia that confirmed by surgical intervention (Fig. 5). Preserved bowel loops mural enhancement is good prognostic sign of reversible bowel ischemia that approved by medical follow up for patients without need for surgical intervention. Four patients showing associated bowel dilatation (Fig. 5). All patients showing mesenteric vessels filling defects. (Fig. 5). 


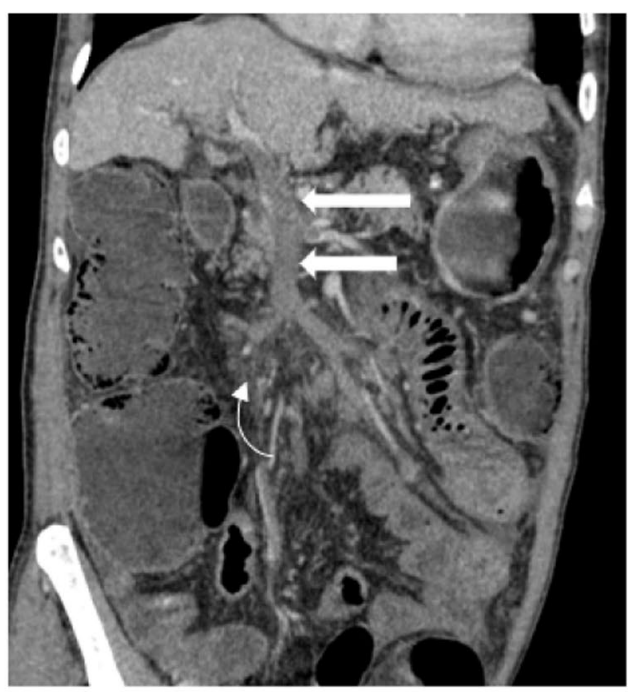

(A)

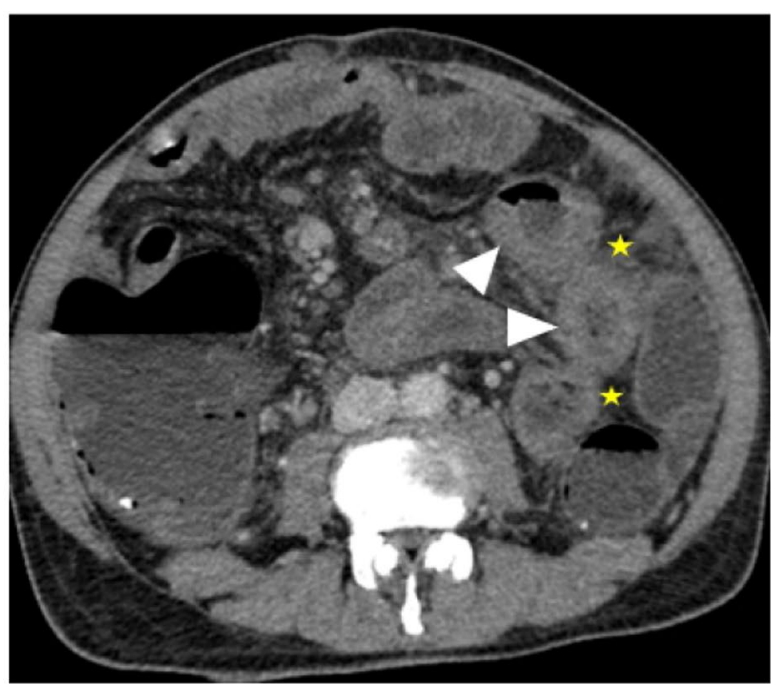

(B)

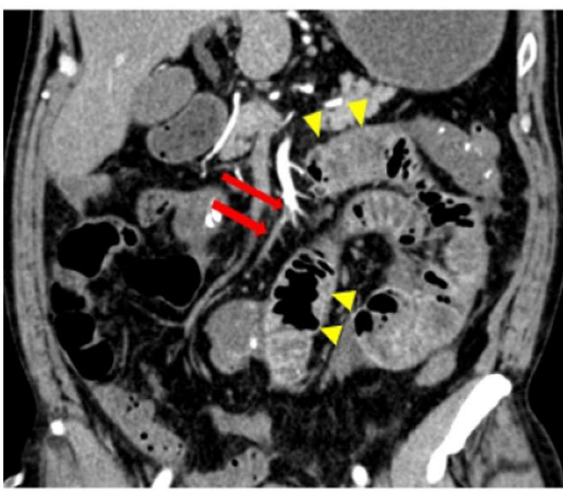

(C)

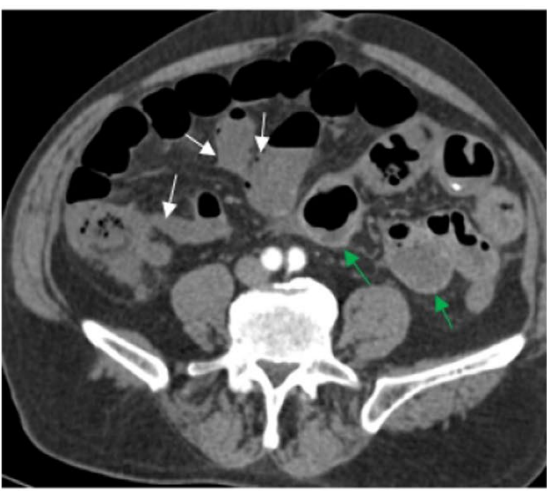

(D)

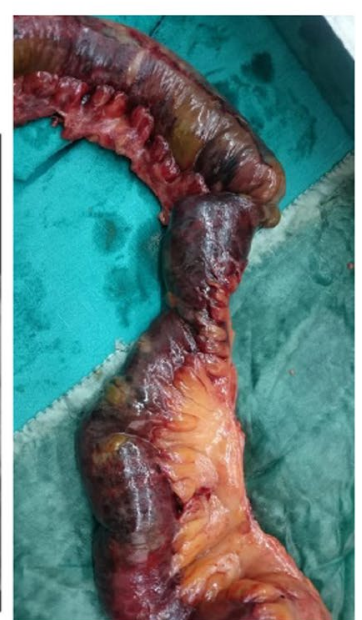

(E)

Fig. 5 A, B For a 64-year-old male patient diagnosed with superior mesenteric vein occlusion. C-E For a 72 -year-old male patient diagnosed with superior mesenteric artery occlusion. A Coronal oblique $\mathrm{CT}$ in venous phase showing complete occlusion of the superior mesenteric and portal veins (white arrows) as well as fat stranding around the mesenteric vein (curved white arrow). B Axial CT in venous phase showing mural thickening (white arrow heads) with preserved mural enhancement associated with mild surrounding fat stranding (yellow star). C Coronal CT in arterial phase showing filling defect at the superior mesenteric artery and one of its branches (red arrows) with dilated small bowel loops (yellow arrow heads). D Axial CT in arterial phase showing mural hypo-enhancement of ileal bowel loops (white arrows) compared to normal enhancement of the jejunal loops (green arrows). E Image of resected ischemic bowel loop that approve the CT diagnosis of bowel ischemia

- Perforated small bowel:

Four patients diagnosed with small bowel perforation: acute abdominal pain is the main presenting symptom followed by vomiting.

The most common frequent CT findings in cases with perforated small bowel is pneumoperitoneum (Fig. 6) that show sub-phrenic distribution in two patients and mesenteric distribution in two patients. Mural thickening of the affected bowel with surrounding fat stranding and free fluid collection (Fig. 6) that seen in three patients. One patient with duodenal perforation is showing associated related abscess formation (Fig. 7) which managed surgically and resolved in follow up imaging. Only one patient with history of trauma showing hypo-enhancement of the perforated bowel. 


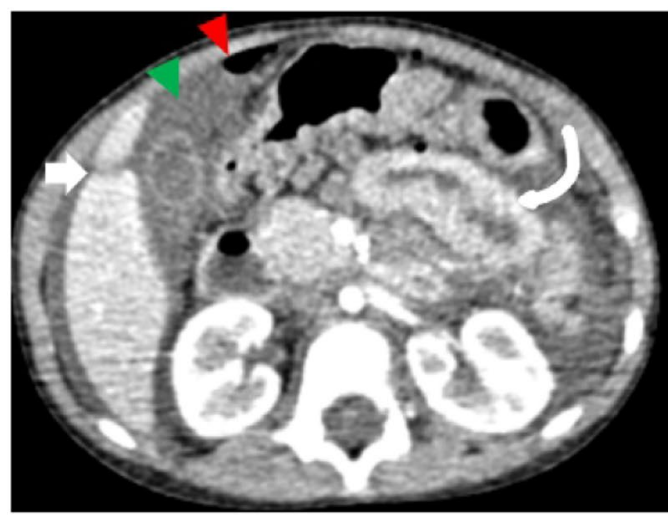

(A)

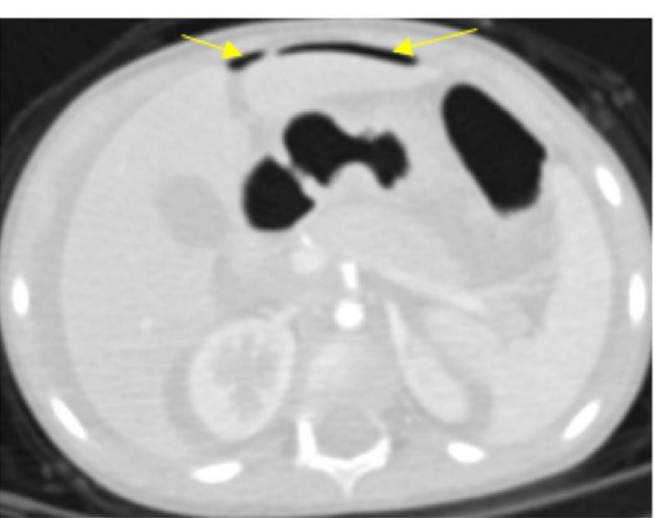

(B)

Fig. 6 A, B For a 4-year-old female patient diagnosed with traumatic bowel perforation. A Axial view in arterial phase showing pneumoperitoneum (red arrow head), free intra-peritoneal fluid collection (green arrow head), hepatic laceration (white arrow) and mild mural thickening of the proximal jejunal loops (curved white arrow). B Axial view in arterial phase (lung window) showing air foci between the liver and anterior abdominal wall (yellow arrows)

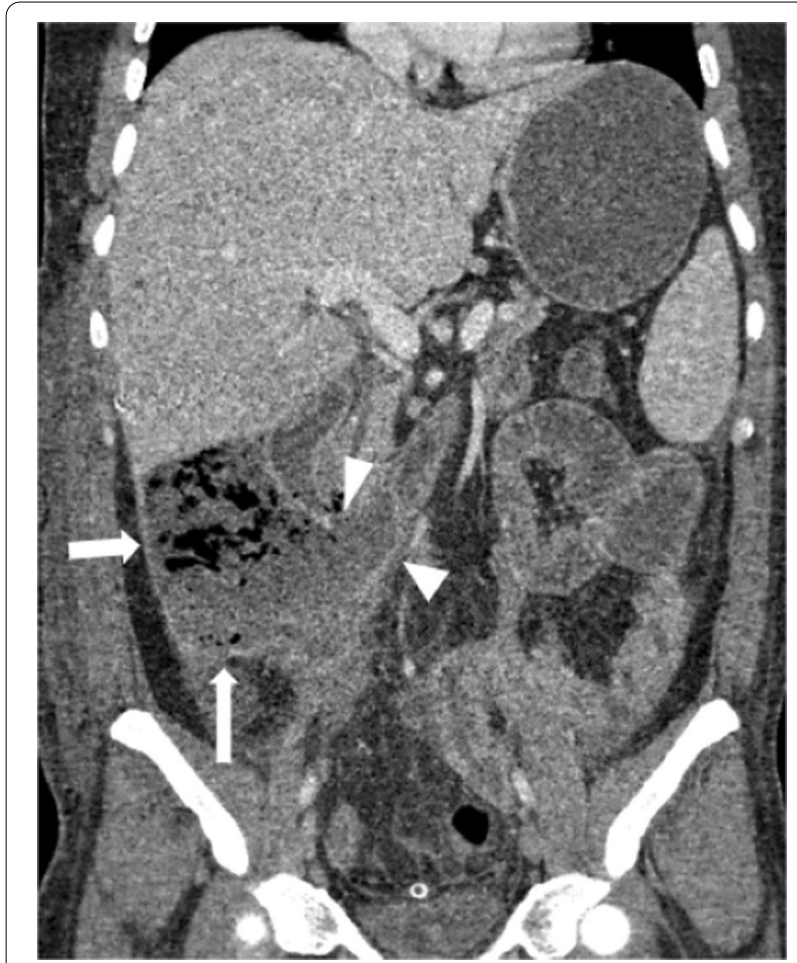

Fig. 7 A 43-year-old male patient diagnosed with perforated duodenal ulcer. Coronal view in venous phase showing abscess formation (white arrow) with related defect at the junction between the second and third part of duodenum (white arrow heads)

\section{Discussion}

Cross-sectional $\mathrm{CT}$ is used in assessment of acute small bowel diseases due to its advantages over the conventional imaging modalities; it assesses the small bowel lumen and wall, surrounding mesentery as well as other abdominal organs. CT is a useful imaging modality in acute setting. Also, the high spatial resolution of CT making it a good modality for achieving better anatomical details [9].

The current study was carried out on 38 patients presented clinically with acute abdominal pain secondary to already known or suspected small bowel origin. There were 23 male and 15 females. The age of involved patients ranges from 4 to 88 years with mean age of incidence about 48 years. No specific considerations regarding the age and sex.

The studied patients are diagnosed as the following: 13 patients with history of pathologically proved inflammatory bowel disease, 12 patients diagnosed with small bowel obstruction, eight patients diagnosed with mesenteric vascular occlusion, four patients diagnosed with perforated bowel and one patient diagnosed with specific small bowel infection (TB).

The inflammatory bowel diseases are the most frequent cause of acute abdominal pain secondary to small bowel origin which agreed by Bassiouny et al. [10] and Shokralla et al. [11].

Our results stated that the small bowel obstruction was the second frequent cause of acute abdominal pain secondary to small bowel origin followed by mesenteric vascular occlusion and infectious bowel disease, respectively, which in agreement with Bassiouny et al. [10] and in disagreement with Shokralla et al. [11] who stated that the mesenteric vascular occlusion is the second frequent cause of acute abdominal pain secondary 
to small bowel origin. Different sample size of involved patients may contribute in such disagreement.

CT entero-colonography showing signs of active inflammatory bowel changes in 12 patients and only one patient is missed by CTE giving about $92.3 \%$ sensitivity of CTE in detection of acute inflammatory changes in patients with history of pathologically proved Crohn's disease.

The high sensitivity of CTE in our study (more than 90\%) in detection of Crohn's disease acute inflammatory signs was matched with Furukawa et al. [12] and Santos et al. [13] who stated the sensitivity of CTE is over 90\%, while Sharma et al. [14], Haas et al. [15] and Monib et al. [16] stated lower sensitivity of CTE (less than 90\%) about $83-86 \%$ which is lower than our results. This explained by different sample size and wide variety of included small bowel pathologies in our study as Sharma et al. [14] studied the difference imaging features between the Crohn's disease and intestinal tuberculosis, while Haas et al. [15] and Monib et al. [16] studied only the imaging features of Crohn's disease.

Mural thickening, mucosal hyper-enhancement and submucosal edema coupled with surrounding fat stranding were the most frequent inflammatory signs seen among patients with Crohn's disease that denoting acute exacerbation. This is in confirmed by Kilcoyne et al. [8], Athanasakos et al. [3] and Monib et al. [16].

Skip lesions followed by Comb sign, enlarged mesenteric lymph nodes, inflammatory stricture, abscess formation and finally fistulae formation, respectively, were the second frequent signs seen among the Crohn's disease patients after aforementioned most common signs, Monib et al. [16] stated that. However, our results are in disagreement with Monib et al. [16], the second frequent signs among our results respectively are Comb signs, enlarged lymph nodes, fistulae formation, skip lesions, abscess formation and finally inflammatory stricture. Such mismatching may explained by different sample size.

The current study showed that the most common involved part of small bowel by Crohn's disease is ileum (83.3\%) followed by combined involvement of ileum and duodenum as well as ileum and jejunum which are equally presented (8.3\%). This is more or less in agreement with Kilcoyne A et al. [8] and Santos et al. [13].

Raman et al. [17] and Monib et al. [16] stated that bilateral symmetrical sacroiliitis is the most common extra-intestinal osseous manifestations of Crohn's disease. This matching with our results in that two patients among the examined cases are suffering from bilateral sacroiliitis which is the only noted osseous finding of Crohn's disease.
Bassiouny et al. [10] stated that TB enteritis is the most frequent cause of infectious small bowel disease which more or less matching with our results, as the only included patient suffering from infectious small bowel disease diagnosed by TB enteritis.

MDCT plays an important role in diagnosis and differentiation between different diseases that affect the small bowel. The CT features of the examined patient showed homogeneous mural enhancement of the terminal ileum and cecum without mural stratification coupled with characteristic necrotic lymph nodes, mild free intra-peritoneal fluid collection and lung involvement. Those signs achieved by usage of CT help in differentiating TB enteritis from Crohn's disease that usually affect the terminal ileum. Mural stratification pattern of enhancement, skip lesions, multi-segmental involvement of small bowel, frequent associated fistulae and abscess formation with the absence of free intra-peritoneal fluid collection that in favor with Crohn's disease. This is confirmed by Sharma et al. [14].

Twelve patients among the examined patients are diagnosed with intestinal obstruction, only one of them diagnosed with adhesive intestinal obstruction managed by follow up that show total resolution of the symptoms after one day of treatment. The others are successfully diagnosed by CECT which reflecting high sensitivity of CT in the diagnosis of IO. Silva et al. [18], Elsayed et al. [19] and Paulson et al. [5] are matching with our results as they stated that MDCT has high sensitivity to diagnose small bowel obstruction reaching up to $100 \%$.

The main diagnostic feature of mechanical intestinal obstruction is small bowel dilatation of the obstructed bowel loops $(>2.5 \mathrm{~cm})$ with distal collapse loops is which is seen in all examined patients. Mian et al. [20], Silva et al. [18] and Millet et al. [21] stated that the dilation of small bowel obstruction is the main diagnostic criterion in diagnosis of small bowel obstruction which is matching with our study.

Searching for the site of transition zone helps in identification of the cause of obstruction. Adhesive intestinal obstruction representing the most frequent cause of small bowel obstruction among the examined patients (58.3\%) than obstructed hernia. This is in agreement with Mian et al. [20], Paulson et al. [5], Silva et al. [18], Taydas et al. [22] and Elsayed et al. [19] as the increasing rate of operative interference as well as early diagnosis and management of hernia help in making the adhesive small bowel obstruction more frequent cause of small bowel obstruction rather than hernia.

Previous surgical operation plays an important role in development of adhesive bands as post-operative sequence which leading to small bowel obstruction. There are seven patients diagnosed with adhesive 
intestinal obstruction; five patients $(71.5 \%)$ have previous abdominal surgical intervention, while two patients have no previous surgical history. Mian et al. [20] and Elsayed et al. [19] stated that post-operative adhesions are responsible of adhesive small bowel obstruction in $70 \%$ and $75 \%$ of their examined patients, respectively, which is matching with our results.

Other causes of small bowel obstruction through our studied patients are obstructed hernia, intussusception and gall stone ileus which are equally represented. This is in disagreement with Paulson et al. [5] and Taydas et al. [22] who stated that hernia and small bowel cancer are the second frequent causes of small bowel obstruction, while intussusception and gall stone ileus are less common. Our study included most of causes of acute abdomen secondary to small bowel origin which affect the sample size of the examined obstructed patients. This may affect our results and leading to such disagreement.

Silva et al. [18] stated that pneumobilia, ectopic gall stone and small bowel obstruction are the diagnostic triad of gall stone ileus which is matching with our result as one of our patients diagnosed with gall stone ileus due the presence of three important CT signs; pneumobilia, ectopic gall stone and dilated small bowel.

Through our study, CT shows high sensitivity of the diagnosis of mesenteric bowel ischemia which confirmed by surgical intervention in $100 \%$ of patients. Kanasaki et al. [2], Moschetta et al. [23] and Furukawa et al. [24] who stated mild lower sensitivity of $\mathrm{CT}$ in assessment of mesenteric ischemia averaging $90-96 \%$, this mismatching may referred to different sample size and different studied patient as in our study we involve other cause of acute small bowel diseases.

Acute mesenteric ischemia due to venous thrombosis was the most frequent cause of small bowel ischemia among the examined patients, while the arterial occlusion was the second frequent cause. This is in disagreement with Kanasaki et al. [2], Moschetta et al. [23] Furukawa et al. [24] and Sugi et al. [1].

While Hefny et al. [25] and Wong et al. [26] are matching with our results as they stated that superior mesenteric vein thrombosis is more frequent than superior mesenteric artery occlusion. We attributed the high frequency of superior mesenteric vein thrombosis in comparison with superior mesenteric artery occlusion through the studied cases to associated liver cirrhosis in MVT patients as predisposing factor of thrombosis which seen in three patients while seen only in one patient diagnosed with superior mesenteric artery occlusion. Also, sample size may contribute in such disagreement.

Florim et al. [27], Kanasaki et al. [2], Copin et al. [28] and Furukawa et al. [24] stated that mural thickening is the most frequent small bowel finding in patients diagnosed with acute mesenteric ischemia, while other noted finding such as mesenteric fat stranding, dilated bowel loops and absent mural enhancement are associated with ischemic bowel diseases but with lower frequencies. This is in agreement with our results as the mural thickening was the most frequent small bowel finding.

Mesenteric vessel filling defect noticed in all examined patients which is a high sensitive CT sign for diagnoses of acute mesenteric ischemia. This is in agreement with Florim et al. [27] and Kanasaki et al. [2].

The perforated peptic ulcer was the most common cause of perforated small bowel disease representing about (50\%) of examined patients. This is matching with Kim et al. [29], Furukawa et al. [30] and Pouli et al. [31].

Traumatic bowel perforation is the second frequent cause of small bowel perforation representing about (25\%) of examined patients. Pouli et al. [31] agree with our results as he stated that traumatic small bowel perforation is the second frequent cause of small bowel perforation, while Kim et al. [29] and Furukawa et al. [30] stated that necrotic and ulcerated malignancies are the second frequent cause of small bowel perforation. This mismatching with our results may explained by small sample size and wide variations of acute small bowel pathologies among our studies patients.

Pneumoperitoneum was the most frequent sign seen among the examined cases as it helps in identification of the site of perforation. The examined cases having perforated peptic ulcer showing foci of pneumoperitoneum closely related to the site of perforation. This is in agreement with Kim et al. [29], Furukawa et al. [30] and Pouli et al. [31].

The distribution of pneumoperitoneum through the abdomen is seen at the sub-phrenic space in two patients including the porta-hepatis in one of them as well as seen scattered through the mesentery in two patients which help in identification of the site of perforation. The associated free intra-peritoneal fluid collection as well as abscess formation close to the site of perforation beside the pneumoperitoneum help also in identification of the site of perforation. This is matching with $\mathrm{H}$. Toprak et al. [32], Kim et al. [29] and Pouli et al. [31].

\section{Limitations}

Limitations were encountered in our study:

Wide variations of included pathologies (inflammatory small bowel diseases, SB obstruction, mesenteric ischemia and small bowel perforation) which affect the sample size in each pathological entity.

Larger number of patients may be of value in achieving better statistical analysis. 


\section{Conclusions}

This study stated that MDCT is a very accurate imaging modality in the diagnosis and differentiation between acute small bowel diseases that cause abdominal pain secondary to small bowel origin. MDCT can diagnose and evaluate the extension of acute exacerbation of inflammatory small bowel diseases and assess the related complication. Also, can identify the site, level and cause of obstructive small bowel diseases. MDCT help in identification of the cause of mesenteric vascular occlusion either arterial or venous and identify the extension of the small bowel ischemic process. MDCT help in identification of the site of perforation and associated related complications.

\section{Abbreviations}

MDCT: Multidetector computed tomography; IBD: Inflammatory bowel disease; TB enteritis: Tuberculous enteritis; SMA: Superior mesenteric artery; SMV: Superior mesenteric vein; kVp: Kilovoltage peak; mAs: Milliampere-seconds.

\section{Acknowledgements}

Not applicable.

\section{Authors' contributions}

$D E$ : editing the draft and revision of the final manuscript. KM: revision of the final manuscript GA: writing the clinical section. AA: writing the draft. All authors read and approved the final manuscript.

\section{Funding}

Not applicable.

\section{Availability of data and material}

The datasets used and/or analyzed during the current study are available from the corresponding author on reasonable request.

\section{Declarations}

\section{Ethics approval and consent to participate}

Approval for this retrospective study was obtained from the Research Ethics Committee of Alexandria Faculty of Medicine (Ethics committee's reference number: 0106116, IRB No: 00012098, FWA No: 00018699). All study procedures were carried out in accordance with the Declaration of Helsinki regarding research involving human subjects. Written informed consent was obtained from the patients.

\section{Consent for publication}

Consent for the use of patients' data and medical record was obtained.

\section{Competing interests}

The authors declare that they have no competing interests.

\section{Author details}

${ }^{1}$ Department of Radiodiagnosis, Faculty of Medicine, University of Alexandria, Alexandria, Egypt. ${ }^{2}$ Department of General Surgery, Faculty of Medicine, University of Alexandria, Alexandria, Egypt.

Received: 19 April 2021 Accepted: 10 September 2021

Published online: 28 September 2021

\section{References}

1. Sugi MD, Menias CO, Lubner MG, Bhalla S, Mellnick VM, Kwon MH et al (2018) CT findings of acute small-bowel entities. Radiographics 38(5):1352-1369
2. Kanasaki S, Furukawa A, Fumoto K, Hamanaka Y, Ota S, Hirose T et al (2018) Acute mesenteric ischemia: multidetector CT findings and endovascular management. Radiographics 38(3):945-961

3. Athanasakos A, Mazioti A, Economopoulos N, Kontopoulou C, Stathis G, Filippiadis D et al (2015) Inflammatory bowel disease-the role of crosssectional imaging techniques in the investigation of the small bowel. Insights Imaging 6(1):73-83

4. Xing GS, Wang S, Sun YM, Yuan Z, Zhao XM, Zhou CW (2015) Small bowel stromal tumors: different clinicopathologic and computed tomography features in various anatomic sites. PLoS ONE. 10(12):e0144277

5. Paulson EK, Thompson WM (2015) Review of small-bowel obstruction: the diagnosis and when to worry. Radiology 275(2):332-342

6. Wells ML, Fletcher JG, Froemming AT, Barlow JM, Fidler JL, Hansel SL et al (2018) CT for evaluation of acute gastrointestinal bleeding. Radiographics 38(4):1089-1107

7. Puylaert CAJ, Tielbeek JAW, Bipat S, Stoker J (2015) Grading of Crohn's disease activity using CT, MRI, US and scintigraphy: a meta-analysis. Eur Radiol 25(11):3295-3313

8. Kilcoyne A (2016) Inflammatory bowel disease imaging: current practice and future directions. World J Gastroenterol:WJG 22(3):917-932

9. Sinha R (2018) Intestinal imaging: the last frontier. J Gastrointest Abdom Radiol 01(01):007-011

10. Bassiouny RH, Khattab RT (2018) Acute non traumatic abdominal pain of small bowel origin: can multi-detector CT enterography provide a potential contribution to the diagnosis of underlying causes? Egypt J Radiol Nucl Med 49(4):934-947

11. Shokralla SY, Amin FG (2020) Non-traumatic abdominal pain: assessment of diagnostic value of MDCT enterography in small bowel diseases-a retrospective study. Egypt J Radiol Nucl Med 51(1):1-9

12 Furukawa A, Saotome T, Yamasaki M, Maeda K, Nitta N, Takahashi M et al (2004) Cross-sectional imaging in Crohn disease. Radiographics 24(3):689-702

13 Santos CHMD, Menezes JNDS, Nunes TF, Martins LDA (2015) CT enterography in the evaluation of Crohn's disease. J Coloproctol (Rio de Janeiro) 35(4):217-22

14. Sharma R, Madhusudhan KS, Ahuja V (2016) Intestinal tuberculosis versus crohn's disease: clinical and radiological recommendations. Indian J Radiol Imaging 26(2):161-172

15. Haas K, Rubesova E, Bass D (2016) Role of imaging in the evaluation of inflammatory bowel disease: how much is too much? World J Radiol 8(2):124-131

16. Monib AM, Abu Gamrah SH, Bayomi AM (2015) Role of multi-detector CT in assessment of patients suspected with chronic inflammatory bowel disease. Egypt J Hosp Med 59(59):182-190

17. Raman SP, Horton KM, Fishman EK (2013) Computed tomography of Crohn's disease: the role of three dimensional technique. World J Radiol 5(5):193-201

18. Silva AC, Pimenta M, Guimaraes LS (2009) Small bowel obstruction: what to look for. RadioGraphics 29(2):423-439

19. Elsayed E, Habib R, Soltan B (2018) Role of multidetector computed tomography in the diagnosis of intestinal obstruction. Menoufia Med J 31(4):1456-1462

20. Mian M, Swamy N, Angtuaco T (2019) Imaging in acute intestinal obstruction. Contemp Diagn Radiol 42(24):1-7

21. Millet I, Ruyer A, Alili C, Curros Doyon F, Molinari N, Pages E et al (2014) Adhesive small-bowel obstruction: value of CT in identifying findings associated with the effectiveness of nonsurgical treatment. Radiology 273(2):425-432

22. Taydaş O, Ünal E, Onur MR, Akpınar E (2018) Role of computed tomography in intestinal obstruction. Istanb Med J 19(2):105-112

23. Moschetta M, Telegrafo M, Rella L, lanora A, Angelelli G (2014) Multidetector $C T$ features of acute intestinal ischemia and their prognostic correlations. World J Radiol: WJR 6(5):130-138

24. Furukawa A, Kanasaki S, Kono N, Wakamiya M, Tanaka T, Takahashi M et al (2009) CT diagnosis of acute mesenteric ischemia from various causes. Am J Roentgenol 192(2):408-416

25. Hefny AF, Ahmed I, Branicki FJ, Ramadan K, Czechowski J, Abu-Zidan FM (2008) Management of mesenteric vascular occlusion. Singap Med J 49(4):316-319

26 Wong Y-C et al (2013) Mesenteric vascular occlusion: comparison of ancillary CT findings between arterial and venous occlusions and 
independent CT findings suggesting life-threatening events. Korean J Radiol 14(1):38-44

27. Florim S, Almeida A, Rocha D, Portugal P (2018) Acute mesenteric ischaemia: a pictorial review. Insights Imaging 9(5):673-682

28. Copin P, Purcell Y, Vilgrain V, Ronot M, Copin P, Nuzzo A et al (2018) Acute mesenteric ischemia: a critical role for the radiologist. Daign Interv Imaging 99(3):123-134

29. Kim SH, Shin SS, Jeong YY, Heo SH, Kim JW, Kang HK (2009) Gastrointestinal tract perforation: MDCT findings according to the perforation sites. Korean J Radiol 10(1):63-70

30. Furukawa A, Sakoda M, Yamasaki M, Kono N, Tanaka T, Nitta N et al (2005) Gastrointestinal tract perforation: CT diagnosis of presence, site, and cause. Abdom Imaging 30(5):524-534
31. Pouli S, Kozana A, Papakitsou I, Daskalogiannaki M, Raissaki M (2020) Gastrointestinal perforation: clinical and MDCT clues for identification of aetiology. Insights Imaging 11(1):1-19

32. Toprak H, Yilmaz T, Yurtsever İ, Sharifov R, Gültekin M, Yiğman S et al (2019) Multidetector CT findings in gastrointestinal tract perforation that can help prediction of perforation site accurately. Clin Radiol 74(9):736. e1-736.e7

\section{Publisher's Note}

Springer Nature remains neutral with regard to jurisdictional claims in published maps and institutional affiliations.

\section{Submit your manuscript to a SpringerOpen ${ }^{\circ}$ journal and benefit from:}

- Convenient online submission

- Rigorous peer review

- Open access: articles freely available online

- High visibility within the field

- Retaining the copyright to your article

Submit your next manuscript at $\boldsymbol{\nabla}$ springeropen.com 